\title{
Emploi des exercices interactifs via l'internet pour remédier aux erreurs d'orthographe en FLE auprès des étudiants de la faculté de pédagogie de Minia
}

\author{
Mohamed Gomaa Refaï Ibrahim \\ Maître de conférences en didactique du FLE \\ Faculté de pédagogie - Université de Minia \\ mgomaa660@gmail.com
}

\section{Résumé :}

Les erreurs d'orthographe sont l'une des difficultés épineuses qui attirent constamment l'attention des chercheurs, car l'écriture est l'un des indicateurs du niveau des compétences linguistiques des apprenants. Cette recherche a visé à remédier aux erreurs d'orthographe en FLE auprès des étudiants de deuxième année de la Faculté de Pédagogie de l'Université de Minia. Afin d'atteindre ce but, les erreurs d'orthographe de ces étudiants ont été identifiées au moyen de trois textes de dictée. L'analyse des erreurs d'orthographe commises par las participants dans les trois textes de dictée a montré que les erreurs les plus communes des participants étaient : les erreurs à dominante phonétique : 202 erreurs $=50 \%$, les erreurs à dominante morphogrammique grammaticale : lettres finales muettes : 87 erreurs $(21.5 \%)$, les erreurs à dominante phonogrammique (l'oral est correct, mais l'écrit est erroné) : 57 erreurs (14\%), les erreurs concernant les homophones grammaticaux (ou encore logogrammes): 43 erreurs $(10.6 \%)$ et les erreurs concernant les homophones lexicaux (ou encore logogrammes): 15 erreurs (3.7\%). Afin de remédier à ces erreurs, le chercheur a utilisé les exercices interactifs via l'internet (des exercices sur les graphies des sons, des exercices interactifs pour trouver les erreurs, des exercices interactifs pour trouver et corriger les erreurs, des exercices interactifs sur les homophones, les lettres muettes et les lettres doubles, le $\mathrm{H}$ muet et le $\mathrm{H}$ aspiré, la conjugaison des verbes, l'accord du participe passé, le pluriel du nom, les pronoms relatifs, l'adjectif verbal ou participe présent, les prépositions et l'orthographe des phrases). L'expérimentation a été exécutée auprès d'un échantillon expérimental composé de 35 étudiants de $2^{\text {ème }}$ année de la Faculté de pédagogie de l'Université de Minia, au cours du 
premier semestre de l'année universitaire 2020/2021. Les résultats de l'expérimentation ont indiqué qu'il y a des différences statistiquement significatives entre les moyennes des notes des participants dans la pré et la post application du test d'orthographe en FLE en faveur de la poste application. Ces résultats ont montré l'efficacité des exercices interactifs via l'internet dans la remédiation aux erreurs d'orthographe en FLE. Par conséquent, la recherche actuelle recommande l'utilisation des exercices interactifs via l'internet pour remédier aux erreurs d'orthographe en langue française.

Mots clés : Exercices interactifs, Erreurs d'orthographe, Français langue étrangère 


\section{استخدام التهمارين التفاعلية عبر الانترنت لعلاج أخطاء الكتابة الإهلائية}

\section{بالاغة الفرنسية كلغة أجنبية لدى طلاب كاية التزبية جاهعة المنيا}

اعـــلـاد

د محمد جمعة رفاعي إبراهييم

مدرس المناهج وطرق تلدريس اللفة الفرنسية

كلية التربية - جامعة المنيا

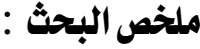

الأخطاء الإملائية من القضايا الثائكة التي تسترعي انتباه الباحثين بصورة مستمرة، حيث

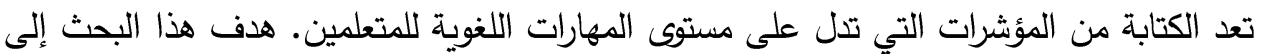

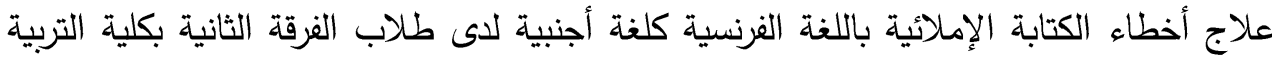

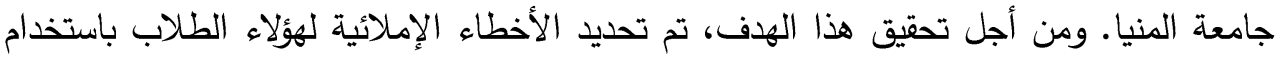

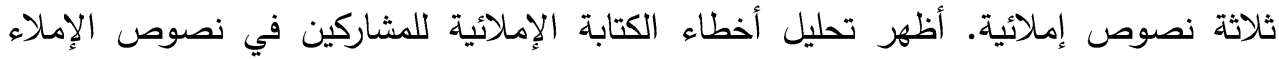

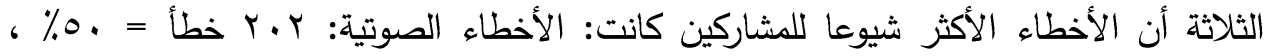

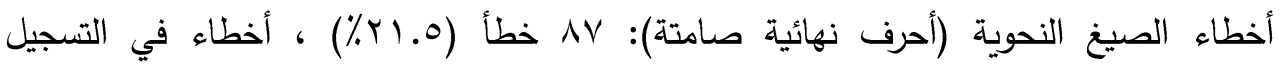

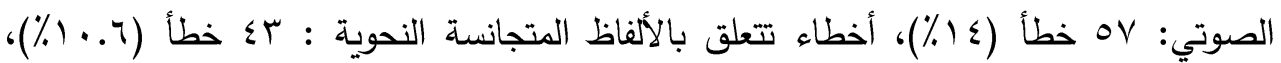

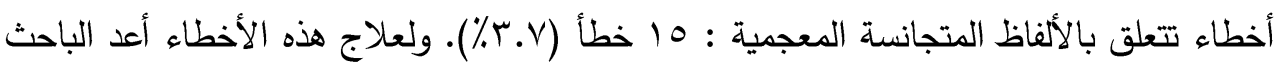

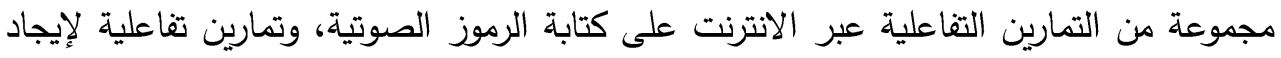

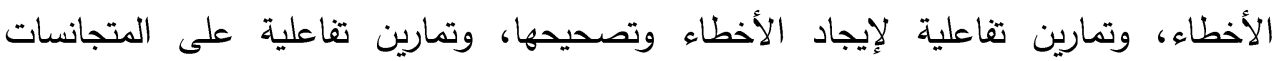

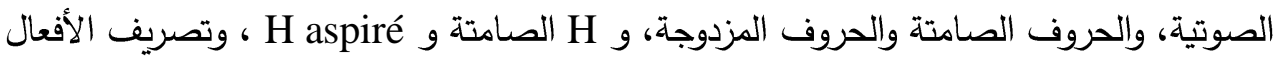

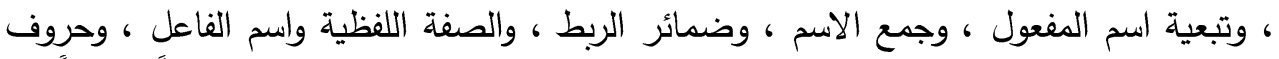

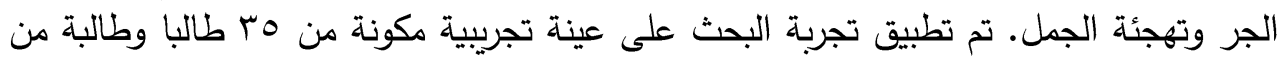

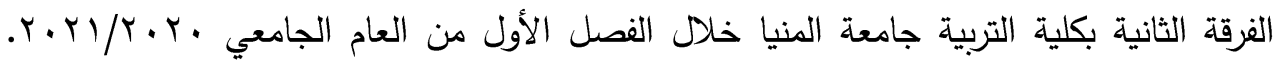

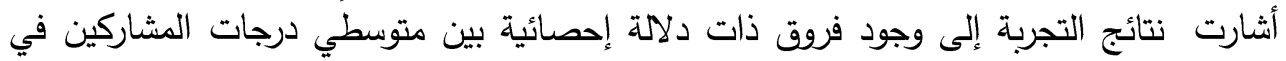

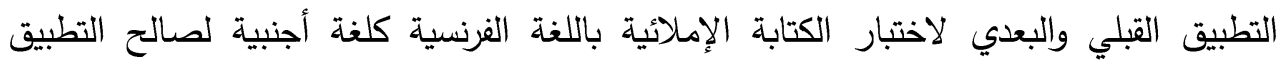
البعدي. أظهرت هذه النتائج فاعلية التمارين التقاعلية عبر الانترنت في علاج الإنة أخطاء الكتابة

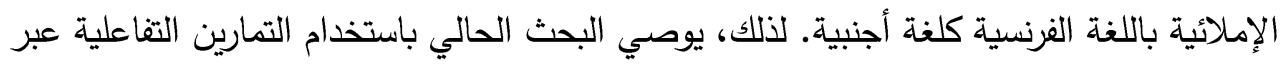

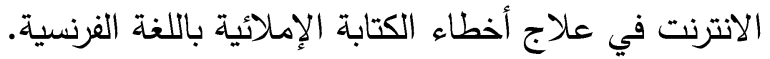
الكلمات المفتاحية: التمارين التفاعلية، الأخطاء الاملائية، اللغة الفرنة الفرنية الفية كلغة أجنبية 


\section{Introduction :}

L'orthographe est d'une importance primordiale dans l'acquisition des langues et surtout les langues étrangères. Elle est un moyen d'apprendre la lecture et l'écriture et même les compétences orales. De même, l'orthographe peut être considérée comme indicateur du niveau de l'étudiant dans les compétences langagières; pour cette raison, elle peut être considérée comme des processus d'améliorer les compétences langagières, ainsi qu'un résultat des processus et des exercices langagiers. Une bonne connaissance de l'orthographe permet aux apprenants non seulement d'écrire correctement, mais aussi, elle a une influence positive sur leur performance et leurs compétences dans les autres activités de la langue.

Shahar-Yames et Share (2008) ont émis l'hypothèse que l'orthographe conduirait à des niveaux significatifs (c'est-à-dire supérieurs au hasard) d'apprentissage de la langue; l'orthographe entraînerait en fait un meilleur apprentissage par rapport à la lecture en raison des exigences de traitement supplémentaires invoquées lors de l'orthographe.

Il est à noter que l'orthographe, en français, est complexe et difficile sur le plan lexical autant que sur le plan grammatical. Pour cette raison, l'apprentissage de connaissances suffisantes pour bien écrire et pour bien comprendre un texte requiert un enseignement régulier sur ce plan tout au long du parcours scolaire (Couzon, 2018). La complexité de 1'orthographe du français est attribuable à ses marques inaudibles à l'oral, notamment grammaticales. Elle (l'orthographe du français) est considérée comme l'une des plus complexes au monde (Bosse, 2019).

Dans la plupart des cas, les apprenants ont des difficultés pour apprendre l'orthographe du français. L'apprentissage des règles ne suffit pas à assurer la maîtrise de la langue. Par conséquent, il faut commencer par comprendre l'origine de ces difficultés. Ensuite, on doit dresser un panorama des connaissances sur le fonctionnement de l'orthographe et sur les questions que pose son apprentissage, qu'il devient possible d'améliorer les performances des apprenants (Fayol et Jaffré, 2014). 
La difficulté de l'orthographe est l'une des difficultés les plus compliquées rencontrées par les étudiants en français langue étrangère. Ce problème est un phénomène qui mérite d'être étudié et abordé, afin d'identifier leurs manifestations et leurs causes, et de déterminer quelles sont les méthodes de remédiation les plus appropriées pour les surmonter afin que l'apprenant puisse poursuivre sa progression et sa réussite scolaire.

Pour développer l'orthographe et remédier à ses erreurs, plusieurs stratégies ont été utilisées par les études antérieures ; l'étude de Morenval, (2015) a utilisé la carte mentale pour remédier aux difficultés de l'orthographe chez les élèves du primaire. En fait, cela les amène à manipuler le langage d'une manière différente et l'étude des homophones ont / on et de l'accord dans la phrase nominale étaient l'objet de cette étude. L'auteur a utilisé la carte mentale pendant la dictée et pendant la correction, qui était soit individuelle, soit en groupes de trois ou quatre. La carte mentale permet d'organiser plus facilement les critères en orthographe et cette présentation particulière est plus compréhensible pour les élèves de cet âge. Petit à petit, cet outil développe des automatismes chez les élèves en orthographe grammaticale et renforce la mémorisation des élèves.

L'étude de Le Brun et al (2016) a mesuré l'efficacité d'un entrainement basé sur les dictées guidées (chaque mot du texte est à écrire dans un «squelette » composé de cases indiquant son nombre de lettres, leur forme et la présence de « graphèmes » qui représentent plusieurs lettres associées en un seul son ou «phonème ») par un support de plusieurs indices sur les mots à écrire, surtout le nombre de lettres. Cet entrainement est comparé à un entrainement par la dictée classique. Les résultats ont montré que l'entrainement avec la dictée guidée est plus efficace que l'entrainement avec la dictée classique pour les élèves de niveau moyen-faible en orthographe. Les élèves très faibles en orthographe progressent de façon similaire quel que soit le type d'entrainement (dictée guidée ou dictée classique) et aucun progrès n'est observé chez les élèves déjà performants en orthographe. 
De même, l'étude de Bosse (2019) a montré que grâce à la dictée guidée, les élèves « faibles mais pas trop » ont pu mobiliser des règles orthographiques qu'ils avaient du mal à solliciter spontanément. Pour ces élèves, le guidage faciliterait la réflexion explicite sur leurs productions écrites.

L'étude de Mohamed (2020) a élaboré des ateliers de négociation graphique dans le développement de quelques compétences orthographiques chez les élèves de sixième primaire dans l'une des écoles francophones à Alexandrie. Afin de collecter les données, la chercheuse a administré un pré-post-test sous la forme d'une dictée portant sur l'accord du participe passé. L'analyse des données a indiqué l'efficacité de la négociation graphique dans l'étude le développement des compétences orthographiques chez les élèves des écoles primaires francophones.

Pour remédier aux erreurs d'orthographe, il est meilleur de travailler l'orthographe dans un climat de confiance, sans peur de l'erreur. Les exercices interactifs en ligne présentent un type d'activités où les apprenants se sentent plus confiants car ils envisagent un système électronique et pas humain. De même, ils peuvent refaire l'exercice et diminuer le taux d'erreurs commises. La correction automatique permet une rétroaction immédiate sur les travaux des apprenants ; ce qui les encourage à déployer plus d'efforts pour remédier aux erreurs d'orthographe.

Souvent, à l'ère des TIC, les étudiants en savent plus sur les ordinateurs que leurs enseignants. Les étudiants ont ainsi poursuivi des domaines d'intérêt à des niveaux auxquels ils deviennent plus compétents et polyvalents que leurs enseignants. Par conséquent, l'idée que les enseignants sont des fontaines de connaissances et que les étudiants sont des récipients vides attendant d'être remplis des connaissances et de la sagesse de leurs enseignants est intenable à l'ère de l'information. La quantité de connaissances disponibles, leur ampleur et leur profondeur dépassent de loin le domaine des seuls enseignants. Leur contrôle de l'accès des apprenants aux connaissances diminue. Le rôle de l'enseignant dans un environnement TIC doit nécessairement changer pour aider les 
étudiants à apprendre de la meilleure façon possible en reconnaissant les besoins et les capacités des apprenants différents et en guidant les étudiants dans leurs choix sur la manière et le lieu d'accéder aux nouvelles connaissances. Le rôle de l'enseignant est donc important dans les processus d'apprentissage. Les enseignants doivent être plus adaptables; plus ouverts aux approches d'enseignement alternatives (Teye, 2011).

Les exercices interactifs en ligne sont un outil par excellence d'entraînement, d'autoévaluation, d'enrichissement à l'apprenant. Ces exercices mis à la disposition des apprenants permettent de s'exercer, de vérifier sa compréhension, de dépister ses lacunes et donc l'amener à combler ces dernières et évoluer vers la maîtrise du sujet visé. Le rôle majeur des exercices interactifs en ligne est essentiellement l'autoévaluation des apprentissages. C'est l'apprenant qui répond aux questions, note ses erreurs, corrige, reprend l'exercice et répond de nouveau aux questions, note à nouveau les erreurs résiduelles et ainsi de suite jusqu'à ce qu'il réussisse le test. Les exercices interactifs en ligne offrent à l'apprenant une évaluation formative. L'exercice fait, donc, partie de l'apprentissage. Ce type d'outils met, alors, l'apprenant face à ses lacunes et l'amène à faire l'effort de chercher les informations qui lui manquent (Bouheraoua, 2006).

"A partir de l'autoévaluation, l'apprenant devrait être capable de conduire son processus d'apprentissage d'une manière autonome. L'évaluation formatrice est une procédure qui vise à rendre l'apprenant compétent en matière d'autoévaluation et d'autocorrection (apprendre à apprendre) » (Ibrahim, 2016, p. 56).

De même, les différentes ressources sont disponibles à l'apprenant, elles mettent à sa disposition toutes sortes d'informations de tous les types et dans toutes les formes. L'apprenant peut adapter ses façons de recevoir l'information, de l'intégrer, de l'enrichir, etc... Il déploie l'effort à penser son sujet, à penser les informations dont il a besoin, à utiliser les moteurs de recherche pour trouver les informations, faire la synthèse, assimiler, approfondir, etc... De cette manière, l'acquisition est intellectuellement plus active. Par conséquent, L'apprenant, en quelque sorte, est son propre formateur. 
L'apprenant motivé est bien plus proactif en ligne que face à un enseignant en classe ou face à un document écrit. Il est appelé à développer de nouvelles habiletés intellectuelles dans l'acquisition des connaissances, il est de plus souhaitable qu'il sache manipuler et utiliser tous les supports de représentation de l'information et surtout de faire les liens parmi les informations dispensées par ces divers supports (Bouheraoua, 2006).

L'étude d'El Hilali et al (2019), dans un projet mené au Québec dans des classes du primaire et du secondaire (482 élèves), a mesuré les effets des dictées métalinguistiques-interactives (la phrase dictée $d u$ jour et la dictée zéro faute) sur la compétence des élèves à orthographier les homophones grammaticaux. Les résultats de cette étude ont montré que les dictées métacognitives-interactives ont un impact positif et significatif sur la compétence des élèves à orthographier les homophones dès le primaire.

L'étude de Salem et Soleiman (2013) a utilisé les exercices interactifs d'autoévaluation pour développer l'autonomie des apprenants de FLE et la compréhension des cours de méthodologie de français. Les résultats de cette étude ont montré que les exercices interactifs influencent positivement l'autonomie des apprenants de FLE ainsi que leur compréhension de cours de méthodologie de français. Ces résultats ont montré, également, que cette influence est plus positive en cas du type de feedback surtout si ce dernier est accompagné par la possibilité de communiquer en ligne avec l'enseignant.

Tuesday et Temidayo (2014) ont cité que l'interaction est centrale aux ressources pédagogiques fournies par l'Internet lors qu'il s'agit de l'apprentissage des langues étrangères. L'apprentissage ludique et la simulation permettent une interaction entre l'apprentissage et les ressources pédagogiques. Pour ce qui concerne l'autonomisation, l'apprenant recherche l'information authentique et s'exprime librement devant son ordinateur. Pour ces raisons, les deux auteurs ont recommandé l'intégration des processus de la théorie d'interactionnisme médiatisée par l'Internet dans l'enseignement/apprentissage des langues étrangères en général et de 
la grammaire du FLE en particulier dans les milieux non francophones.

\section{Problématique de la recherche :}

Le problème des erreurs d'orthographe reste encore une question digne à discuter et à traiter. Le chercheur a observé pendant l'enseignement aux étudiants de la faculté de pédagogie, université de Minia que les étudiants commettent beaucoup d'erreurs dans leurs écritures en FLE. Ce problème est vérifié à travers les travaux écrits présentés par les étudiants. Ils font, constamment, des erreurs d'orthographe lexicale : lettres muettes, lettres doubles, homophones, ainsi que des erreures d'orthographe grammaticale : conjugaison des verbes, accord du participe passé, pronoms relatifs. De même, cette problématique est assurée par les études antérieures (Salem et Soleiman, 2014 ; Gaafar, 2019). Salem et Soleiman (2014) ont indiqué que les étudiants licenciés ès Lettres, et ceux ès Lettres et Pédagogie - Département du français à l'Université de Mansourah n'arrivent pas à écrire correctement et que leur niveau orthographique est extrêmement bas. Ces licenciés ont un problème avec les différentes composantes d'orthographe et surtout celles du type lexical. Pour envisager ce problème, la recherche actuelle va répondre aux questions suivantes :

1. Quelles sont les erreurs communes de l'orthographe en FLE auprès des étudiants de la $2^{\text {ème }}$ année de la faculté de pédagogie de Minia?

2. Quels sont les exercices interactifs via l'internet pour remédier aux erreurs communes de l'orthographe en FLE auprès des étudiants de la $2^{\text {ème }}$ année de la faculté de pédagogie de Minia?

3. Quelle est l'efficacité des exercices interactifs via l'internet pour remédier aux erreurs communes de l'orthographe en FLE auprès des étudiants de la $2^{\text {ème }}$ année de la faculté de pédagogie de Minia? 


\section{Objectifs de la recherche :}

1. Déterminer une liste d'erreurs communes de l'orthographe en FLE auprès des étudiants de la $2^{\text {ème }}$ année de la faculté de pédagogie de Minia ;

2. Classifier les erreurs commises par les étudiants de la $2^{\text {ème }}$ année de la faculté de pédagogie de Minia selon la fréquence pour traiter les erreurs les plus communes ;

3. Remédier aux erreurs communes de l'orthographe en FLE auprès des étudiants de la $2^{\text {ème }}$ année de la faculté de pédagogie de Minia ;

4. Vérifier l'efficacité des exercices interactifs via l'internet pour remédier aux erreurs communes de l'orthographe en FLE auprès des étudiants de la $2^{\text {ème }}$ année de la faculté de pédagogie de Minia.

\section{Délimitation de la recherche :}

Cette recherche s'est délimitée à :

1. Un groupe de recherche composé de 35 étudiants de la $2^{\text {ème }}$ année de la faculté de pédagogie de Minia (section de français) car ils ont, d'une part, suivi une étude spécialisée en français pendant une année et pour pouvoir continuer à développer leurs compétences pendant les années ultérieures, d'autre part.

2. Quelques erreurs de l'orthographe lexicale et grammaticale en FLE les plus communes auprès des étudiants de la $2^{\text {ème }}$ année de la faculté de pédagogie de Minia.

\section{Hypothèses de la recherche :}

1. Il y a une différence statistiquement significative entre la moyenne des notes des étudiants du groupe expérimental au pré-post/test d'orthographe lexicale en FLE chez les étudiants de la $2^{\text {ème }}$ année de la faculté de pédagogie de Minia en faveur du post/test.

2. Il y a une différence statistiquement significative entre la moyenne des notes des étudiants du groupe expérimental au pré-post/test d'orthographe grammaticale en FLE chez les 
étudiants de la $2^{\text {ème }}$ année de la faculté de pédagogie de Minia en faveur du post/test.

3. Il y a une différence statistiquement significative entre la moyenne des notes des étudiants du groupe expérimental au pré-post/test d'orthographe lexicale et grammaticale (total) en FLE chez les étudiants de la $2^{\text {ème }}$ année de la faculté de pédagogie de Minia en faveur du post/test.

\section{Outils de la recherche :}

1. Un test diagnostique pour déterminer les erreurs communes d'orthographe en FLE auprès des étudiants de la $2^{\text {ème }}$ année de la faculté de pédagogie de Minia ;

2. Un pré-post/test d'orthographe en FLE en vue de mesurer l'efficacité des exercices interactifs via l'internet pour remédier aux erreurs communes de l'orthographe en FLE auprès des étudiants de la $2^{\text {ème }}$ année de la faculté de pédagogie de Minia ;

\section{Groupe de la recherche :}

Cette recherche s'est délimitée à un seul groupe expérimental (35 étudiants et étudiantes) parmi les étudiants de la $2^{\text {ème }}$ année de la faculté de pédagogie de Minia car ils ont, d'une part, suivi une étude spécialisée en français pendant une année et pour pouvoir continuer à développer leurs compétences pendant les années ultérieures d'autre part.

\section{Méthodologie de la recherche :}

Afin d'atteindre les objectifs de la recherche, le chercheur utilise une méthode qualitative descriptive pour faire l'analyse des erreurs d'orthographe en FLE auprès des étudiants visés. Pour vérifier l'efficacité des exercices interactifs via l'internet, le chercheur utilise la méthode expérimentale avec un design quasi-expérimental ayant une pré/post évaluation et un seul groupe expérimental. 


\section{Terminologie de l'étude :}

\section{Erreurs d'orthographe :}

Les erreurs d'orthographe sont la transcription des signes écrits contrairement aux normes de la langue ou l'absence des normes orthographiques dans l'écriture. (Définition opérationnelle)

\section{Exercices interactifs :}

Les exercices interactifs sont les exercices en ligne autocorrigés qui présentent une rétroaction simple ou formulée. (Définition opérationnelle)

\section{Cadre conceptuel :}

\section{L'orthographe en français et ses erreurs :}

Le mot orthographe est d'origine grecque (orthographia) qui se compose de deux mots : ortho et graphia qui veulent dire écrire correctement (Catach, 2011). Par conséquent, l'orthographe peut être définie comme la manière d'écrire correctement selon des normes définies dans une langue.

Selon Catach (1995, p.16), l'orthographe est la manière d'écrire les sons ou les mots d'une langue en conformité avec le système de transcription graphique adopté à une époque donnée en suivant certains rapports avec les sous-systèmes de la langue (morphologie, syntaxe, lexique). Plus ces rapports sont complexes, plus le rôle de l'orthographe grandit car un tissu d'antagonismes se crée entre les relations phonie-graphie et les autres considérations qui entrent en ligne de compte. L'orthographe est un choix entre ces diverses considérations, ce choix est, plus ou moins, réglé par des lois ou des conventions diverses.

Pour Fayol et Jaffré (2014, p.11), l'orthographe est la représentation graphique et conventionnelle d'une langue donnée. Elle utilise des procédés originaux dont les principes relèvent d'une forme quasi universelle d'écriture. Elle doit obéir à des contraintes diverses qui peuvent être dictées par la structure linguistique et par des contextes sociaux qui en font une norme et parfois, un objet culturel. 
Le Dictionnaire La Rousse (en ligne) définit l'orthographe ainsi : "Ensemble de règles et d'usages définis comme norme pour écrire les mots d'une langue donnée. (On distingue l'orthographe d'accord, fondée sur les règles de la grammaire, et l'orthographe d'usage, qui n'obéit pas à des règles précises.) 》

Traditionnellement, on peut distinguer " l'orthographe d'usage, ou absolue, qui concerne les mots tels qu'on les trouve écrits dans un dictionnaire, sans égard à leur fonction dans la phrase, et l'orthographe de règle, ou d'accord, qui prend en compte les rapports que les mots d'une phrase ont entre eux conformément aux règles de la grammaire »(Demnard, 1981, p.593).

La description de Catach (1980; dans Sautot, 2002) du système orthographique a tenté de rendre compte de toute la substance graphique du français. Selon cette description, les différents graphèmes sont classés selon quatre critères : la fréquence, la cohésion, le rapport direct avec le phonème, et la rentabilité. Le critère de fréquence est plus intéressant sur le plan pédagogique car il indique la priorité. La rentabilité pédagogique indique qu'il faut d'abord s'intéresser aux parties les plus stables du système graphique. Le critère de rentabilité s'appuie sur le lien d'ordre sémantique ou grammatical que le graphème crée. Par exemple, le graphème EAU de "chevreau" ne représente que 3\% des réalisations graphiques du phonème [o] mais sa rentabilité est importante car il permet la création des mots dérivés : chèvre / chevreau ... Il en est de même pour le graphème $\mathrm{AI}$ peu rentable dans la création lexicale mais dont l'importance est majeure dans la conjugaison à l'imparfait et au conditionnel. Le critère de cohésion permet de délimiter les graphèmes complexes, composés de plusieurs lettres. Il permet, également, de déterminer la frontière de certains graphèmes composés de plusieurs lettres. Si une unité graphique perd le contact avec sa réalisation orale, elle tend à sortir du système. Ainsi le P de "temps" permet de faire "temporel". Il reste donc une certaine relation avec l'oral puisque ce $\mathrm{P}$ est clignotant : parfois il est prononcé. De même, dans le verbe, le $\mathrm{S}$ de la première personne du pluriel "nous avons", est systématiquement associé au phonogramme ON qui le 
précède. Il y a encore contact avec l'oral. En revanche, le $\mathrm{S}$ de "souris" est définitivement détaché de l'oral. La proximité de l'oral charge donc le graphème de sens. Plus il est proche de l'oral, plus le graphème contribue à véhiculer du sens. Plus il s'en éloigne, moins il en véhicule. Une unité totalement détachée de l'oral est une lettre "morte" du point de vue sémantique. Ces quatre critères permettent un classement des divers graphèmes en grandes catégories. La principale catégorie qui représente environ $80 \%$ des lettres écrites est la catégorie des phonogrammes. Vient ensuite la catégorie des morphogrammes, puis les catégories marginales du système, les logogrammes, lettres historiques et lettres doubles.

Le principe fondamental à l'œuvre dans l'écriture alphabétique $\mathrm{du}$ français est le principe phonogrammique, qui met en correspondance les unités de l'écrit avec les unités non significatives de l'oral. Ce principe se double en français d'un second principe : le principe morphogrammique qui décrit le fait que certaines unités de l'écrit (appelées morphonogrammes) sont en rapport avec des unités pourvues de sens lexical et grammatical : les morphèmes. L'orthographe du français constitue un ensemble organisé et cohérent, un ensemble de systèmes et chacun des systèmes correspond à un principe d'écriture ainsi : (Mout, 2013).

1. Le principe phonogrammique selon lequel les unités de l'écrit notent les unités non significatives de l'oral (les graphèmes $\mathrm{p}$ et a dans papa notent les phonèmes /p/et /a/) ;

2. le principe morphogrammique selon lequel les unités de l'écrit donnent des informations grammaticales et des informations lexicales qui peuvent être absentes du système oral (par exemple, les graphèmes $s$ (marque grammaticale) et $t$ (marque lexicale) dans les enfants) ;

3. le principe distinctif ou logogrammique selon lequel l'orthographe du français distingue des mots homophones, établissant ainsi un rapport de type sémiographique entre le signifiant écrit et le signifié (c'est ainsi qu'on distingue a de à, pause de pose). 
Mout (2013) présente un autre principe lié à ces trois précédents : ce sont certaines lettres étymologiques ou historiques qui ne jouent plus de rôle dans le système graphique d'aujourd'hui. Il s'agit en particulier des consonnes doubles purement graphiques, de lettres latines, et de lettres grecques.

Le système orthographique complexe de la langue française rend sa maitrise difficile surtout chez les apprenants arabophones. Par conséquent, plusieurs études ont été faites sur les erreurs d'orthographe et leurs classifications. Asselin et McLaughlin (1992) ont relevé les erreurs dans la rédaction des étudiants universitaires pour créer des instruments d'évaluation et d'amélioration de la compétence en français écrit. Soixante étudiants ont rédigé plusieurs textes sur divers thèmes imposés. Après avoir analysé les erreurs commises par les étudiants, les chercheuses ont classé ces erreurs selon six catégories (syntaxe, grammaire, ponctuation, orthographe lexicale, lexique et sémantique et coupure de mots en fin de ligne).

Parmi les classifications les plus importantes des erreurs d'orthographe en français est la classification de Catach (1980) qui classifie les erreurs d'orthographe selon six catégories ainsi :

\section{- Erreurs à dominante phonétique :}

Ce type d'erreurs est dû à une mauvaise production orale : manmam pour maman, à cause d'une ignorance de la prononciation correcte. Pour remédier à ces erreurs, il faut assurer la connaissance précise des différents phonèmes.

\section{- Erreurs à dominante phonogrammique :}

Dans ce type d'erreurs, l'oral est correct, mais l'écrit est erroné. Le phonogramme est un graphème qui est chargé de transcrire de différents phonèmes : ã correspond à plusieurs phonogrammes (graphèmes) en, em, an, am. Par exemple o pour eau. Ces erreurs peuvent être provisoires avant le passage à une orthographe correcte.

\section{- Erreurs à dominante morphogrammique :}

Les morphogrammes sont des graphèmes non chargés de transcrire des phonèmes : des marques finales de liaisons (finale muette d'un mot), des marques grammaticales (morphogrammes de genre, morphogrammes de nombre $s, x$, morphogrammes verbaux $e$, 
$s, e$ ), marques finales de dérivation (grand - grandeur) et marques internes de dérivation (main - manuel). Les erreurs à dominante morphogrammique sont lexicales ou grammaticales (dans le cas des erreurs à dominante morphogrammique de type grammaticale, les erreurs portent sur les accords et ne relèvent pas d'une logique immuable).

\section{- Erreurs concernant les homophones (logogrammes) :}

Ces erreurs peuvent être lexicaux (chant / champ) ou grammaticaux (c'est /s'est). Ils peuvent, également, relever du discours.

\section{- Erreurs concernant les idéogrammes :}

Tout signe qui ne relève pas uniquement de l'alphabet est considéré comme idéogramme. C'est le cas des majuscules, des signes de ponctuation.

\section{- Erreurs concernant les lettres non justifiables d'un enseignement :}

Ces erreurs entre dans les anomalies de la langue française (Nid /nidifier mais abri / abriter).

Pour enseigner l'orthographe et remédier à ses erreurs d'orthographe, les auteurs ont utilisé plusieurs stratégies. Morenval (2015) nous présente trois propositions pour enseigner l'orthographe :

1. Mettre les élèves au centre des apprentissages

Pour que les enseignants envisagent le problème des erreurs d'orthographe auprès les apprenants, ils doivent leur proposer des activités significatives. Ces activités peuvent être enrichies par la présence d'un destinataire car les apprenants écrivent dans un but précis. La contextualisation permet de porter plus de soin et d'attention à l'orthographe.

2. Le traitement de l'erreur

Pour l'orthographe, l'erreur devrait être une source de travail. Dans ce cas, les erreurs ne devraient pas une cause d'une punition ; mais une cause de réflexion. Par conséquent, l'enseignant doit sensibiliser les apprenants à réfléchir dans et sur leurs erreurs.

3. Dispositifs en production d'écrit/ dictée

Ces dispositifs mettent en place des activités permettant aux élèves d'être au centre des apprentissages. Ces différentes séquences 
se centrent autour de l'idée principale de faire des apprenants les véritables acteurs de leur apprentissage en orthographe, en passant principalement par la manipulation. La manipulation proposée par Morenval (2015) se concrétise sous deux formes : la construction personnelle d'une carte mentale en dictée et le recours à des étiquettes constituant des corpus manipulables par les apprenants. De même, ces dispositifs s'appuient sur deux thématiques favorisant l'apprentissage des apprenants : le premier met en valeur le rôle de l'écriture dans l'acquisition des règles orthographiques, et part du principe suivant : « c'est en écrivant qu'on apprend à écrire». Le dispositif consiste à passer par la rédaction de listes pour mettre en application les règles orthographiques. Le second dispositif vise la création et l'appropriation d'un outil orthographique par les apprenants dans le but de les rendre autonomes pour la correction de la dictée.

L'étude de Wang (2014) a examiné les perceptions de 52 étudiants taïwanais sur les exercices interactifs après avoir assisté à des séances d'enseignement en classe et après avoir fait des devoirs en ligne hebdomadaires sous la forme d'exercices interactifs sur le Web pendant un semestre. Les perceptions des apprenants sur les exercices interactifs en ligne ont été obtenues par une enquête informatisée menée à la fin du semestre. Les résultats de l'enquête ont révélé que les participants percevaient les exercices interactifs en ligne comme intéressants, mais modestement. Cela était probablement attribuable au niveau de difficulté des exercices créés et au manque de formats d'exercices divers. Même ainsi, avoir un accès facile, recevoir une rétroaction immédiate, autoriser plusieurs tentatives et permettre l'apprentissage à son propre rythme ont été mentionnés comme des avantages des exercices construits. Plus important encore, la plupart des participants ont signalé l'efficacité de ces exercices pour améliorer leur compréhension de la lecture et leur apprentissage du vocabulaire. Cette évaluation de la langue a confirmé les résultats qui ont démontré des gains significatifs en compréhension de lecture et en connaissance du vocabulaire. Les implications comprennent des suggestions pour les professeurs de langues et les développeurs de programmes pour améliorer la manière dont les activités d'apprentissage en ligne sont créées et mises en œuvre. 


\section{Les exercices interactifs via l'internet :}

La théorie constructiviste présente quelques stratégies d'enseignement/apprentissage qui rendent les apprenants acteurs de leur apprentissage. Les outils 2.0 permettent aux apprenants d'être actifs à travers la participation et la collaboration. Les exerciseurs en ligne (exercices interactifs) sont l'un de ces outils qui donnent aux apprenants l'occasion de vérifier leurs connaissances, de déterminer leurs erreurs, de recevoir une rétroaction immédiate et de refaire l'exercice pour corriger les erreurs commises.

De même, les exercices interactifs en ligne rendent les apprenants plus autonomes. Les résultats de l'étude de Savchuk et al (2019) ont signalé que l'apprentissage interactif a abouti à un changement dans les préférences des apprenants en matière d'apprentissage, y compris un plus grand désir de s'engager de manière autonome dans le travail assisté par ordinateur, une résolution plus rapide des problèmes, une motivation accrue à étudier et une meilleure gestion du temps et des compétences d'apprentissage tout au long de la vie. De plus, il y a eu un changement dans les approches pédagogiques des enseignants, à savoir d'une approche centrée sur l'enseignant à une approche centrée sur l'étudiant.

L'enseignement interactif a commencé par une philosophie de l'enseignement avec la technologie et a abouti à un nouveau processus d'enseignement et d'apprentissage interactif. Une combinaison de constructivisme, de tableaux interactifs et d'outils Web 2.0 est un modèle pour réfléchir à de nouvelles méthodes d'enseignement. Dans ce stade, les étudiants et les enseignants sont au cœur du processus. Les enseignants sont responsables de la planification, de l'enseignement et de la facilitation des séquences intégrées à la technologie. Les étudiants sont responsables de la construction et de la démonstration des connaissances ainsi que de la participation pour créer des connaissances. Au cours de la phase de planification, les enseignants réfléchissent aux outils qui peuvent améliorer l'expansion cognitive des étudiants, comme la mise en œuvre d'outils Web 2.0 qui les aident à accéder et à traiter l'information. Dans la phase d'enseignement interactif, les enseignants modélisent l'utilisation de la 
technologie pour construire des connaissances et démontrer des concepts grâce à des interactions dynamiques (Sessoms, 2008).

La discussion sur les relations entre l'interactivité technique et pédagogique indique une voie à suivre pour se concentrer davantage sur les objectifs d'apprentissage pendant l'activité afin de permettre aux apprenants d'être plus autonomes dans l'exploitation des possibilités des TIC. Dans ce type d'apprentissage, les étudiants apportent des connaissances, des compétences et des dispositions relatives à la matière enseignée. La combinaison des possibilités du milieu et des connaissances des étudiants peut offrir un potentiel d'action pour atteindre les objectifs d'apprentissage. Dans le même temps, les contraintes du cadre peuvent structurer leurs actions sous une forme positive (orientation) ou négative (obstacle à surmonter). (Kennewell et al, 2008).

L'interactivité est le processus de communication réciproque entre le formateur et l'apprenant ou entre les apprenants eux-mêmes dans les différents types de formation à distance. L'interactivité est également définie par l'usage de technologies qui permettent d'avoir une rétroaction. Par exemple, une vidéo de formation pourrait comporter des éléments technologiques qui permettent à l'apprenant de donner son avis ou encore de participer à un sondage (Chovino et Dallaire, 2009).

Une salle de classe interactive est une salle de classe qui facilite les conversations virtuelles et face à face entre les enseignants et les apprenants. C'est un programme dans lequel les apprenants participent en tant que partenaires égaux au processus d'apprentissage. L'enseignant joue le rôle de facilitateur et de guide, mais les apprenants jouent avec enthousiasme leur rôle dans l'apprentissage. L'apprentissage consiste à impliquer les étudiants dans une activité ou une tâche qui incitera l'apprenant à réfléchir et à analyser les informations enseignées. Cela peut se produire à chaque étape ou à chaque niveau d'une leçon, depuis l'engagement des étudiants dans le sujet, en participant activement et consciemment à la découverte du langage et des règles, jusqu'à la production libre et active (Nurtika, without date). 
L'enseignement interactif est conçu autour d'un principe simple : sans application pratique, les étudiants ne parviennent souvent pas à comprendre la profondeur du matériel d'étude. Alors que les étudiants perdent souvent leur intérêt au cours du style d'enseignement traditionnel, les styles d'enseignement interactifs favorisent une atmosphère d'attention et de participation. Ces techniques présentent de multiples avantages : l'instructeur peut facilement et rapidement évaluer si les étudiants ont vraiment maîtrisé le matériel (et prévoit d'y consacrer plus de temps, si nécessaire), et le processus de mesure de la compréhension des élèves dans de nombreux cas est également pratique pour le matériel. Enfin, la nature même de ces évaluations favorise l'interactivité et apporte plusieurs avantages (Nurtika, sans date).

Il est nécessaire de distinguer entre deux types de stratégies psychopédagogiques susceptibles d'augmenter l'interactivité qui consistent tantôt en des interactions entre humains par l'intermédiaire du système, tantôt en une interaction de type homme-machine : (Henry, Vandeput \& Brigitte, 2010)

\section{- Interactions entre humains :}

L'interactivité humaine se retrouve à travers trois modes de communication, à savoir les emails, les forums et les rencontres en présentiel. Quel que soit le mode de communication employé, l'important est de réagir rapidement et régulièrement aux demandes, mais surtout de les stimuler. Un apprenant en difficulté a la possibilité de correspondre par email avec un tuteur. Il peut également interagir via les forums avec d'autres apprenants. Rappelons qu'il a aussi la possibilité de rencontrer une personne-ressource au sein de son établissement scolaire.

\section{- Interactions homme-machine :}

Afin d'éviter toute distance pédagogique, l'apprenant jouit d'une certaine liberté au sein du dispositif. Il est seul responsable de son apprentissage et se voit offrir la possibilité d'effectuer des choix tant sur le plan du contenu, des démarches que des interactions. Il peut, à tout moment, accéder aux activités qu'il souhaite refaire. Il garde la liberté d'effectuer ou non les exercices, de faire des lectures 
supplémentaires. Sa créativité est encouragée à travers des activités où il est appelé à mettre au point sa propre stratégie de résolution d'un problème. Dès qu'il y est arrivé, il peut confronter sa démarche à celle proposée dans le dispositif. Il construit ses connaissances et est amené à les adapter selon les situations rencontrées.

Il est à noter que les exercices interactifs via l'internet reposent sur la stratégie de l'interaction homme-machine ; ces exercices sont automatiquement corrigés et une rétroaction est présentée à l'apprenant. La rétroaction peut être simple qui indique que la réponse est correcte ou non, ou formulée qui présente une information utile dans ce cas. Ce qui rend ces exercices plus importants est la possibilité de refaire l'exercice plusieurs fois ; cet état rend les processus d'apprentissage plus dynamiques et plus flexibles.

Pour le rôle de l'apprenant dans l'environnement du système d'apprentissage interactif, il consiste en une combinaison d'aptitudes et de compétences décrites ci-dessous ; son rôle est structuré dans une combinaison dynamique de cinq compétences opérationnelle, cognitive, collaborative, auto-directionnelle et spécifique au cours : (Teye, 2011)

\section{- Compétence opérationnelle}

L'utilisation efficace du système d'apprentissage interactif comme outil d'apprentissage, de communication, de collaboration et d'auto-direction est un rôle essentiel des apprenants. Les apprenants doivent avoir une expertise adéquate dans l'utilisation des outils TIC pour la communication et la collaboration. Ils doivent également être en mesure d'accéder aux ressources d'informations, de les analyser et de les partager.

\section{- Compétence cognitive}

Les apprenants doivent pouvoir apprendre à partir du contenu du cours, appliquer leurs propres connaissances et demander de l'aide lorsque cela est nécessaire. Les apprenants doivent savoir comment accéder aux supports de cours et s'y prendre pour en tirer le meilleur parti. Les apprenants doivent apprendre de manière réflexive grâce à 
la pensée critique, où les connaissances sont appliquées pour résoudre des problèmes

\section{- Compétence collaborative}

La communication et la collaboration avec les camarades de classe et les enseignants sont l'un des rôles de l'apprenant. Les apprenants doivent être disposés à communiquer et à exprimer leurs idées, opinions et sentiments. Ils doivent également être capables d'impliquer leurs camarades de classe dans l'apprentissage ou dans le travail de groupe, participer aux discussions et aux activités de classe, être prêts à rechercher des commentaires et à en tirer des leçons, et à donner des commentaires afin d'apprendre.

\section{- Compétences spécifiques au cours}

Ces compétences requièrent à l'assimilation efficace ou l'utilisation de terminologies et de processus ou de savoir-faire spécifiques au domaine de connaissances. Celles-ci pourraient inclure l'utilisation du langage et des terminologies de conception de base avec les camarades de classe et l'enseignant, la lecture des contributions des camarades de classe aux discussions et aux activités de la classe, et la prise de note d'expressions et de vocabulaire nouveaux ou intéressants utilisés par l'enseignant ou par les camarades de classe. Cela comprend également la participation d'idées, d'opinions et d'expériences à des discussions en classe.

Quant aux processus de la production et de l'utilisation d'exercices interactifs en ligne, ils s'organisent techniquement autour de trois étapes : La création, la diffusion et la collecte des données (Laigle, 2010)

\section{- La création}

La première étape consiste à créer une batterie de questions et à les organiser en questionnaires. Chaque logiciel propose un choix plus ou moins grand d'exercices (mots-croisé, textes à trous, QCM, dictées, etc.) et d'interactivité (intégration du multimédia, indices, rétroactions...) 


\section{- La diffusion}

Une fois les questionnaires créés, ils peuvent être diffusés et utilisés de différentes façons.

- La façon la plus simple est de déposer les fichiers créés sur l'ordinateur de l'apprenant (via une clé USB par exemple).

- Si l'établissement possède un réseau local, les fichiers peuvent être distribués via l'espace commun accessible sur tous les ordinateurs.

- Les exercices peuvent également être mis en ligne sur Internet. Il est à noter que plusieurs sites présentent la possibilité de créer et mettre en ligne les exercices interactifs sur l'internet.

- Enfin, il est également possible de créer et d'importer les exercices interactifs sur des plateformes e-learning.

\section{- La collecte des données}

Dans cette phase, les apprenants font les exercices interactifs autocorrectifs, une rétroaction leur est adressée. Une occasion de refaire les exercices plusieurs fois est possible.

Pour créer les exercices interactifs en ligne, les formateurs peuvent utiliser plusieurs outils, parmi lesquels, on peut citer LearningApps, Hot potatoes, La Quizinière, Socrative, Educaplay, Kubbu, ... :

LearningApps : c'est un site ou une application qui permet aux enseignants de créer toutes sortes d'exercices interactifs (Apps) en ligne. Dans l'activité ou l'exercice, des images, des documents, des textes, des vidéos, de l'audio peuvent être insérés. Plusieurs activités sont disponibles (QCM, mots-croisés, textes à trous, classement sur un axe, paires, puzzles, ...) (Académie de Poitiers, 2015).

Hot potatoes : C'est un logiciel de création des exercices interactifs en ligne, il se compose de 6 modules : (Laigle, 2010)

- JCloze : pour les textes à trous ;

- JMatch : pour les exercices d'appariement ;

- JCross : pour les mots croisés ;

- JMix : pour les phrases à remettre en ordre ;

- JQuiz : pour les questions à choix multiples ; 
- The Masher : pour rassembler une suite d'exercices au sein d'un questionnaire.

La Quizinière : c'est un outil de création d'activités pédagogiques. Il permet d'accommoder tout type de média et mitonner des exercices pour que les apprenants s'entrainent et révisent en écrivant, en choisissant, en dessinant, en parlant, en chantant... Pour l'accès à un exercice, l'enseignant communique le code aux apprenants ; ceux-ci n'ont pas besoin de créer de compte. L'enseignant peut recevoir leurs copies et leur retourner les corrections (Gilger et Gervais, 2020).

Socrative : c'est une application gratuite en ligne qui permet de réaliser des tests, des quiz pour une utilisation pédagogique. Socrative nécessite que l'enseignant crée un compte sur la plateforme. Pour les apprenants, ils peuvent participer sans avoir besoin d'avoir un compte (Académie de Rennes, 2017).

Educaplay : c'est une application Web 2.0 de partage et de création d'exercices interactifs en ligne (Carte Interactive; Devinette; Compléter ; Mots Croisés ; Dialogue ; Dictée ; Ordonner les Lettres ; Ordonner les Mots; Relier; Mots Mêlés; Test; Collection; Présentation ; Videoquiz) (PragmaTICE, site internet).

\section{Méthodologie :}

Cette recherche a visé à remédier aux erreurs communes de l'orthographe en FLE chez les étudiants de la $2^{\text {ème }}$ année de la faculté de pédagogie de Minia. Pour atteindre ce but, le chercheur a déterminé les erreurs communes d'orthographe en FLE chez les étudiants de la $2^{\text {ème }}$ année de la faculté de pédagogie à travers test diagnostique composé de trois textes de dictée. Ensuite, il a préparé et a collecté un groupe d'exercices interactifs en ligne et pour mesurer l'efficacité de ces exercices interactifs, le chercheur a élaboré un prépost/test.

\section{Test diagnostique :}

Pour diagnostiquer les erreurs d'orthographe en FLE auprès des étudiants de $2^{\text {ème }}$ année de la faculté de pédagogie de Minia, le chercheur a choisi trois textes de dictée qui sont décrits ainsi (annexe 1) : 
Texte 1. Une Terre sans frontières : 11 phrases ;

Texte 2. Le sang de la Terre : 8 phrases ;

Texte 3. Lettre à une amie : 14 phrases.

\section{Source des textes de dictée :}

La Fondation Paul Gérin-Lajoie (2013). Recueil de dictées. https://fondationpgl.ca/wp-content/uploads/2019/01/Recueil-dedicte\%CC\%81es.pdf

\section{Validité et fiabilité du test :}

Une étude pilote, a été menée pour évaluer la validité et la fiabilité du test. Pour déterminer la validité du test, le chercheur a présenté le test à un jury pour juger l'adéquation des textes au niveau des étudiants de deuxième année de la faculté de pédagogie de Minia. Les membres du jury ont indiqué que les textes sont adéquats pour le niveau des étudiants.

De même, le chercheur a vérifié la validité du test par le calcul des corrélations question-total. Les corrélations des questions-total sont présentées ainsi

$1^{\text {ier }}$ texte : 0.56

$2^{\text {ème }}$ texte : 0.48

$3^{\text {ème }}$ texte : 0.62

De ces résultats, le test diagnostique est valide et peut être untilisé pour diagnostiquer les erreurs d'othographe.

Pour calculer la fidélité du test, le chercheur a administré le test deux fois le 23 févreir 2020, puis deux semaines après. Le chercheur a calculé le coefficient de corrélation entre les deux passations en appliquant la formule statistique (de Person) suivante :

$$
r=\frac{\mathrm{n} \sum \mathrm{xy}-\left(\sum \mathrm{x}\right)\left(\sum \mathrm{y}\right)}{\sqrt{\left(\mathrm{n} \sum \mathrm{x} 2-\left(\sum \mathrm{x}\right) 2-\left(\mathrm{n} \sum \mathrm{y} 2-\left(\sum \mathrm{y}\right) 2\right.\right.}}
$$

$\mathrm{R}=$ coefficient de corrélation

$\mathrm{N}=$ nombre des étudiants

$\mathrm{T}=$ total des notes des étudiants

$\mathrm{X}=$ notes de la $1^{\text {ère }}$ passation

$\mathrm{Y}=$ notes de la $2^{\text {ème }}$ passation 
Le résultat de cette équation a démontré que le coefficient de corrélation entre les deux passations du test est 0.78. Ce résultat affirme que notre test est fidèle.

\section{Résultats du test diagnostique :}

Nous avons administré le test diagostique auprès d'un échantillon des étudiants de la $2^{\text {ème }}$ année de la faculté de pédagogie de Minia (40 étudiants et étudiantes) pendant le premier semestre de l'année universitaire 2020/2021 (le 25 octobre 2020). Les résultats du diagnostic ont démontré que les étudiants de la $2^{\text {ème }}$ année de la faculté de pédagogie de Minia font beaucoup d'erreurs d'orthographe ; le tableau suivant présente les types de ces erreurs.

Tableau 1 : résultats du test diagnostique

\begin{tabular}{llcccc}
\hline $\mathrm{N}^{\circ}$ & Types d'erreurs & $\begin{array}{l}\text { Nombre } \\
\text { des erreurs }\end{array}$ & $\begin{array}{l}\text { Pourcen- } \\
\text { tage }\end{array}$ & $\begin{array}{l}\text { Nombre } \\
\text { des } \\
\text { étudiants }\end{array}$ & $\begin{array}{l}\text { Pourcen- } \\
\text { tage }\end{array}$ \\
\hline 1 & $\begin{array}{l}\text { Erreurs à dominante } \\
\text { phonétique }\end{array}$ & 202 & $50 \%$ & 34 & $85 \%$ \\
\hline 2 & $\begin{array}{l}\text { Erreurs à dominante } \\
\text { morphogrammique } \\
\text { grammaticale (lettres } \\
\text { finales muettes) }\end{array}$ & 87 & $21.5 \%$ & 28 & $70 \%$ \\
\hline 3 & $\begin{array}{l}\text { Erreurs à dominante } \\
\text { phonogrammique } \\
\text { (l'oral est correct, } \\
\text { mais l'écrit est erroné }\end{array}$ & 57 & $14 \%$ & 28 & $70 \%$ \\
\hline 4 & $\begin{array}{l}\text { Erreurs concernant } \\
\text { les homophones } \\
\text { grammaticaux }\end{array}$ & 43 & $10.6 \%$ & 22 & $55 \%$ \\
\hline 5 & $\begin{array}{l}\text { Erreurs concernant } \\
\text { les homophones } \\
\text { lexicaux }\end{array}$ & 15 & $3.7 \%$ & 12 & $30 \%$ \\
\hline
\end{tabular}

Les résultats du test diagnostique ont montré que les étudiants de la deuxième année de la faculté de pédagogie de Minia commettent beaucoup d'erreurs d'orthographe en français. Ces résultats ont montré que les erreurs à dominante phonétique sont les erreurs les plus communes chez les étudiants visés ; ce type d'erreurs 
représente $50 \%$ des erreurs faites par les participants. On remarque, également, que $85 \%$ des participants font des erreurs à dominante phonétique. Les erreurs à dominante morphogrammique grammaticale (lettres finales muettes) représentent $21.5 \%$ des erreurs faites par les participants. $70 \%$ de ceux-ci font des erreurs à dominante morphogrammique grammaticale. Les erreurs à dominante phonogrammique (l'oral est correct, mais l'écrit est erroné) viennent dans le troisième niveau avec un pourcentage $14 \%$ des erreurs faites par les étudiants qui représentent $70 \%$ des participants. Pour les erreurs concernant les homophones grammaticaux, elles représentent $10.6 \%$ des erreurs faites par les étudiants qui représentent $55 \%$ des participants. Les erreurs les moins faites par les étudiants visés sont les erreurs concernant les homophones lexicaux ; mais le pourcentage des participants qui font ces erreurs est $30 \%$.

\section{Elaboration des exercices interactifs :}

Notre recherche vise à remédier aux erreurs d'orthographe lexicale et grammaticale en FLE auprès des étudiants de la $2^{\text {ème }}$ année de la faculté de pédagogie de Minia. Pour atteindre ce but, nous avons élaboré et collecté un groupe d'exercices interactifs en ligne. Nous avons déterminé l'objectif général des exercices interactifs, les objectifs opérationnels, le contenu, les outils, les ressources des exercices interactifs :

\section{Objectif général des exercices interactifs}

Les exercices interactifs en ligne élaborés et collectés visent à remédier aux erreurs communes de l'orthographe lexicale et grammaticale en FLE chez les étudiants de la $2^{\mathrm{e}}$ année de la faculté de pédagogie de Minia.

\section{Objectifs opérationnels}

\section{A la fin de l'exécution des exercices interactifs en ligne, les participants doivent être capables de/d':}

- Ecrire correctement les mots avec les accents : aigu, grave, circonflexe ou un tréma ;

- Ecrire correctement les mots avec la cédille ; 
- Ecrire correctement les graphies du son [è] : è, ê, ai, ei, et en fin de mot ;

- Ecrire correctement les graphies du son [o] : o, au, eau, $\hat{\mathbf{o}}$;

- Ecrire correctement les graphies du son [k] : c, q, k, cc, cu, ch, qu ;

- Ecrire correctement les graphies du son [s] : s, ss, c devant e, i ou y, ç, sc et du son $[z]: s, z$;

- Ecrire correctement les graphies du son [g] ;

- Ecrire correctement les graphies du son [j] ;

- Ecrire correctement les graphies des sons [on] : on, om ;

- Ecrire correctement les graphies du son [in] : in, im, ain, aim, ein, un, en ;

- Ecrire correctement les graphies du son [an] : en, em, an, am, aon ;

- Ecrire correctement les graphies du son [ch] : ch, sh, sch ;

- Ecrire correctement les graphies du son [sion] : sion, ssion, tion ;

- Ecrire correctement les graphies du son [f] : f ou ph ;

- Ecrire correctement les graphies du son [ye] : ill ou y ;

- Ecrire correctement les graphies du son [eur] : eur - eure œur ;

- Ecrire correctement les graphies du son [oi] ;

- Déterminer si le mot contient une erreur d'orthographe ;

- Déterminer les erreurs d'orthographe dans un texte simple ;

- Déterminer et corriger les erreurs d'orthographe dans un texte simple ;

- Distinguer les homophones ;

- Ecrire correctement des homophones ;

- Corriger ses erreurs concernant les homophones ;

- Déterminer les lettres muettes finales dans un mot;

- Déterminer les lettres muettes l'intérieur des mots ;

- Ecrire correctement des mots avec des lettres muettes finales ;

- Corriger des erreurs sur les lettres muettes finales ;

- Déterminer les mots contenant une consomme simple ou une consonne double ; 
- Déterminer les erreurs d'orthographe lexicale sur les lettres doubles ;

- Déterminer les mots qui doublent leur dernière lettre au féminin ;

- Déterminer le $\mathrm{H}$ muet et le $\mathrm{H}$ aspiré dans les mots ;

- Faire ou non l'élision et la liaison selon le $\mathrm{H}$ muet ou le H aspiré ;

- Conjuguer correctement les verbes ;

- Faire l'accord du participe passé d'une manière correcte ;

- Déterminer les formes correctes et erronées dans le pluriel ;

- Utiliser correctement les pronoms relatifs simples;

- Utiliser correctement les pronoms relatifs composés ;

- Lier deus propositions avec un pronom relatif ;

- Utiliser correctement le participe présent ;

- Utiliser correctement l'adjectif verbal ;

- Différencier correctement entre le participe présent et l'adjectif verbal ;

- Utiliser correctement les prépositions ;

- Déterminer si la phrase contient une erreur d'orthographe ;

- Ecrire correctement l'orthographe des mots entendus ;

- Écrire un texte court de dictée.

\section{Contenu et sources des exercices interactifs :}

Le chercheur a déterminé les exercices interactifs en ligne adéquats à la remédiation des erreurs d'orthographes diagnostiquées à travers la recherche dans les sites spécialisés dans ce domaine. De même, le chercheur a élaboré quelques exercices à travers le site https://learningapps.org/ pour compléter le groupe d'exercices demandés pour remédier aux erreurs d'orthographe traitées. Le groupe d'activités contiennent ... :

- Des exercices interactifs pour écrire correctement les graphies des sons [è], [o], [k], [s], [z], [g], [j], [on], [in], [an], [ch], [sion], [f], [ye], [eur], [oi]. 
Source :

https://www.francaisfacile.com/exercices/exercice-francais2/exercice-francais-35890.php

- Des exercices interactifs pour trouver les erreurs

Sources :

https://evalquiz.com/jeucommun/finjeu?idTest=1060\&type=motCorr ect

https://evalquiz.com/phrasefaute/jeuphrasesfautes/jeu?idTest=1054 https://evalquiz.com/tests/orthographe

- Des exercices interactifs pour trouver et corriger les erreurs

Sources : https://orthographe.evalquiz.com/phraseErreur/1

https://orthographe.evalquiz.com/phraseErreur/2 https://orthographe.evalquiz.com/phraseErreur/6 https://orthographe.evalquiz.com/phraseErreur/2 https://orthographe.evalquiz.com/phraseErreur/3 https://orthographe.evalquiz.com/phraseErreur/4

\section{- Des exercices interactifs sur les homophones}

Sources:

https://www.ortholud.com/orthographe/homonymes/texte 1.php https://www.francaisfacile.com/exercices/exercice-francais2/exercice-francais-88253.php https://www.francaisfacile.com/cgi2/myexam/voir2r.php?id=19165 https://www.francaisfacile.com/exercices/exercice-francais2/exercice-francais-3026.php https://www.francaisfacile.com/exercices/exercice-francais1/exercice-francais-221.php

https://www.francaisfacile.com/cgi2/myexam/voir2r.php?id=5826 https://www.francaisfacile.com/exercices/exercice-francais2/exercice-francais-74589.php https://www.ccdmd.qc.ca/fr/exercices interactifs/index.cgi?id=1045 \&action=animer 
- Des exercices interactifs sur les lettres muettes et les lettres doubles

Sources:

https://www.francaisfacile.com/exercices/exercice-francais-

2/exercice-francais-11961.php

https://www.tice-education.fr/tous-les-articles-er-ressources/articlesinternet/1038-creer-des-exercices-interactifs-pour-vos-eleves-aveckubbu

https://www.francaisfacile.com/exercices/exercice-francais2/exercice-francais-32997.php

https://leflepourlescurieux.fr/consonnes-doubles-les-10-astuces-pourles-reperer-orthographe-b1/

https://www.letudiant.fr/quiz/culture-generale/orthographe-et-

grammaire/quiz-orthographe-grammaire-les-consonnes-doubles.html

https://learningapps.org/display?v=psbgqpivk20

https://www.ortholud.com/orthographe/feminin/index.php

https://www.ortholud.com/orthographe/feminin/deux.php

\section{- Des exercices interactifs sur le $H$ muet et le $H$ aspiré}

Sources:

https://www.francaisfacile.com/exercices/exercice-francais-

2/exercice-francais-61194.php

https://www.espacefrancais.com/quiz/divers/h_aspire-

h_muet/quiz.htm

https://www.podcastfrancaisfacile.com/podcast/la-lettre-h.html

- Des exercices interactifs sur la conjugaison des verbes

Sources :

https://orthographe.evalquiz.com/verbeConjugaison/1

https://orthographe.evalquiz.com/verbeConjugaison/2

- Des exercices interactifs sur l'accord du participe passé

Sources:

https://learningapps.org/display?v=pfyyid $2 v k 20$ 
- Des exercices interactifs sur le pluriel du nom

Sources:

https://learningapps.org/display? $v=$ ptsmc $5 \mathrm{knt} 20$

https://learningapps.org/display?v=pw1rizvg320

https://learningapps.org/display? $\mathrm{v}=$ pr9ndqrut20

https://www.francaisfacile.com/exercices/exercice-francais-

2/exercice-francais-34713.php

- Des exercices interactifs sur les pronoms relatifs

Sources :

https://www.francaisfacile.com/exercices/exercice-francais2/exercice-francais-31128.php

https://www.francaisfacile.com/exercices/exercice-francais-

2/exercice-francais-41289.php

https://www.francaisfacile.com/exercices/exercice-francais2/exercice-francais-105619.php

- Des exercices interactifs sur l'adjectif verbal et participe présent

Sources :

https://www.francaisfacile.com/exercices/exercice-francais2/exercice-francais-92704.php

https://www.francaisfacile.com/exercices/exercice-francais-

2/exercice-francais-53247.php

https://www.francaisfacile.com/exercices/exercice-francais-

2/exercice-francais-102268.php

- Des exercices interactifs sur les prépositions

Sources :

https://www.francaisfacile.com/exercices/exercice-francais2/exercice-francais-31584.php

https://www.francaisfacile.com/exercices/exercice-francais-

2/exercice-francais-81756.php

https://www.francaisfacile.com/exercices/exercice-francais-

2/exercice-francais-122274.php

- Des exercices interactifs sur l'orthographe des phrases Sources :

https://evalquiz.com/jeucommun/finjeu?idTest=1189\&type=erreurPhrase https://evalquiz.com/jeucommun/finjeu?idTest=1192\&type=erreurPhrase 
- Des exercices interactifs sur la Dictée :

Sources: https://orthographe.evalquiz.com/orthographeMot/1

https://orthographe.evalquiz.com/orthographeMot/2

https://orthographe.evalquiz.com/orthographeMot/3

https://orthographe.evalquiz.com/dictee/1

https://orthographe.evalquiz.com/dictee/2

https://orthographe.evalquiz.com/dictee/3

\section{Elaboration du pré-post/test d'orthographe :}

\section{Objectif du test :}

Le test élaboré a eu pour objectif de mesurer l'efficacité des exercices interactifs via l'internet pour remédier aux erreurs communes de l'orthographe en FLE chez les étudiants de la $2^{\text {ème }}$ année de la faculté de pédagogie de Minia.

\section{Détermination des sources du test :}

Pour préparer le test, le chercheur a compulsé les études antérieures et les ouvrages qui ont porté sur les erreurs de l'orthographe lexicale et grammaticale, ainsi que les sites internet dans ce domaine.

\section{Description du test :}

\section{Notre pré/post-test est composé de deux parties :}

\section{Première partie : l'orthographe lexicale (30 points)}

- Une question à choix multiples sur les graphies des sons : 10 items ;

- Une question à choix multiples sur les homophones : 5 items ;

- Une question qui demande aux participants de compléter les mots avec une lettre finale muette : 5 items ;

- Une question qui demande aux participants de choisir entre le, la ou l' devant les mots selon le $\mathrm{H}$ (muet ou aspiré) : 5 items ;

- Une question qui demande aux participants de donner un synonyme pour les mots donnés (des mots qui contiennent une consonne ou une consonne double) : 5 items. 
Deuxième partie : l'orthographe grammaticale (30 points)

- Une question à choix multiples sur la conjugaison des verbes (5 items),

- Une question qui demande aux participants de donner le participe passé d'un verbe donné (5 items);

- Une question qui demande aux participants de mettre des mots donnés au pluriel (5 items)

- Une question qui demande aux participants de relier les phrases avec un pronom relatif ( 5 items) ;

- Une question qui demande aux participants de mettre les verbes entre parenthèses soit au participe présent, soit comme adjectif verbal (5 items);

- Une question qui demande aux participants de compléter les phrases avec une préposition convenable (5 items).

\section{Validité et fiabilité du test :}

Une étude pilote a été menée pour évaluer la fiabilité du test. Pour déterminer la validité du test, le chercheur utilise le coefficient de Kuder Richardson-20 en appliquant la formule statistique suivante :

$$
r K R 20=\left(\frac{k}{k-1}\right)-\left(\frac{\Sigma p q}{\sigma 2}\right)
$$

- rKR20 = la formule de Kuder-Richardson 20 ;

- $\mathrm{k}=$ le nombre total d'items de test ;

$-\Sigma$ indique la somme ;

- $\mathrm{p}=$ la proportion de candidats ayant réussi à un item ;

- $\mathrm{q}=$ la proportion de candidats qui échouent à un item

- $\sigma 2$ = la variance de l'ensemble du test.

Le résultat obtenu du coefficient de Kuder Richardson-20 est 0,82. C'est un taux satisfaisant par rapport aux objectifs de la recherche.

Pour calculer la fidélité du test, nous avons administré le test deux fois avec un intervalle de deux semaines. Le chercheur a calculé le coefficient de corrélation entre les deux passations en appliquant la formule statistique suivante :

$$
\boldsymbol{r}=\frac{\mathrm{n} \sum \mathrm{xy}-\left(\sum \mathrm{x}\right)\left(\sum \mathrm{y}\right)}{\sqrt{\left(\mathrm{n} \sum \mathrm{x} 2-\left(\sum \mathrm{x}\right) 2-\left(\mathrm{n} \sum \mathrm{y} 2-\left(\sum y\right)^{2}\right.\right.}}
$$


$\mathrm{R}=$ coefficient de corrélation

$\mathrm{N}=$ nombre des étudiants

$\mathrm{T}=$ total des notes des étudiants

$\mathrm{X}=$ notes de la $1^{\text {ère }}$ passation

$\mathrm{Y}=$ notes de la $2^{\text {ème }}$ passation

Le résultat de cette équation a démontré que le coefficient de corrélation entre les deux passations du test est 0.82 . Ce résultat affirme que notre test est fidèle.

\section{L'expérimentation de la recherche :}

L'expérimentation de la recherche est passée par plusieurs étapes :

- Déterminer le design expérimental et sélectionner les participants ;

- Administrer le pré-test ;

- Expérimenter les exercices interactifs ;

- Administrer le post-test ;

- L'analyse des résultats ;

L'expérimentation des exercices interactifs en ligne a eu lieu pendant le premier semestre de l'année universitaire 2020/2021 (pendant les mois de Novembre et Décembre). Une période de 6 semaines (du 15 novembre jusqu'à 27 décembre) a été consacrée à la mise en application de cette expérimentation. Pour expérimenter les exercices interactifs, le chercheur a fait une rencontre avec les participants en présentiel, les objectifs des exercices interactifs, la stratégie qu'on va suivre leur ont été présentés. Pour faciliter la communication avec les participants, un groupe WhatsApp a été créé. Sur ce groupe, le chercheur partageait les objectifs des unités, les liens des exercices interactifs sur l'internet, les démarches à suivre. Après avoir fait les exercices, les participants envoyaient une capture d'écran de leurs appareils pour bien suivre la progression dans la remédiation à leurs erreurs d'orthographe.

\section{Les types de rétroaction dans les exercices interactifs utilisés}

Dans les exercices interactifs en ligne utilisés, les participants reçoivent une rétraction immédiate de deux types : 
- Rétroaction simple : les participants sont informés si leurs réponses sont exactes ou erronées (Vrai/Faux, Bravo/Erreur ...)

Indiquez si la phrase contient une faute, Vous n'avez que 5 secondes pour répondre.

Bravo

SCORE 80

Chacun sur son cahier recopie la phrase corrigé.

\section{Sans faute $\quad$ Avec faute}

Chacun sur son cahier recopie la phrase corrigée. evalquiz.com

Image 1. Capture d'écran d'un exercice interactif

\section{Erreur \\ SCORE 40}

Chacun sur son cahier recopie la phrase corrigé.

\section{Sans faute}

Avec faute

Chacun sur son cahier recopie la phrase corrigée.

Image 2. Capture d'écran d'un exercice interactif

- Rétroaction formulée : les participants peuvent, également, recevoir une rétroaction formulée en présentant la forme correcte ou une description sur leurs réponses. 
1. L'accor d

2. Le fusi I

3. Le cadena s

4. Le brui t

5. Du lar $\underline{t}=\underline{d}$

6. un ban $c r$

7. Lon g

8. Un accro $\underline{\underline{s}}=\underline{C}$

9. Du plom

10. Du hou $x$

1. Le bra s

12. L'estoma C

Image 3. Capture d'écran d'un exercice interactif

- Hurlement : le « $\mathrm{h}$ » est aspiré. On écrit : le hurlement (et non thurlement).

- Hiérarchie : le « $\mathrm{h}$ » est aspiré. On écrit : la hiérarchie (et non l'hiérarehie).

- Haricot : le « $\mathrm{h}$ » est aspiré. On écrit : le haricot prononcé (et non tharieot).

- Hercule : le « $\mathrm{h}$ » est muet. On écrit : l'hercule prononcé [lèr-kul].

- Ahurissement : le « $\mathrm{h}$ » est aspiré.

Attention ! La lettre « $\mathrm{h}$ » est ordinairement aspirée lorsqu'elle se trouve au milieu d'un mot entre deux voyelles comme dans cohue, aheurter, ahan. (Le Dictionnaire de l'Académie française)

- Haut-parleur : le « h » est aspiré. On écrit : le haut-parleur (et non thaut parleur).

- Huguenot, -e : le « h » est aspiré. On écrit : le huguenot (et non thuguenot).

- Hélium : le « $\mathrm{h}$ » est muet. On écrit : l'hélium prononcé [lél-yom]

- Huit : le « $\mathrm{h}$ » est aspiré. On écrit : le huit / le huitième (et non l'huit / thutitième).

- Héros : le « h » est aspiré. On écrit : le héros (et non thérøs).

Attention ! Tous les dérivés de héros sont sans aspiration (leur « $\mathrm{h}$ » est alors muet). Ce sont : héroïde, l'héroïne, hérö̈que, hérö̈quement, héroïsme.

Lisez la page orthographique concernant le « $\mathrm{h}$ » aspiré et le « $\mathrm{h}$ » muet.

$$
\text { Refaire l'exercice }
$$

Image 4. Capture d'écran d'un exercice interactif

\section{Résultats de la recherche :}

Après avoir fini l'expérimentation des exercices interactifs via l'internet et administré un test d'orthographe employé comme pré/post test en FLE sur le groupe de la recherche, le chercheur a fait l'analyse statistique en utilisant le programme statistique SPSS et il 
utilise "T" test pour calculer la moyenne arithmétique, l'écart type et la valeur de "t".

Tableau 2 : Les résultats du pré/post-test d'orthographe lexicale en FLE du groupe expérimental

\begin{tabular}{|c|c|c|c|c|c|c|}
\hline Test & $\mathrm{N}$ & MA & ET & $\mathrm{T}$ & S. ou N.S. & VI \\
\hline Pré-test & \multirow[t]{2}{*}{35} & 9.4571 & 2.69360 & \multirow{2}{*}{$\begin{array}{c}41.62 \\
8\end{array}$} & \multirow{2}{*}{$\begin{array}{c}\text { Significative au } \\
\text { degré } 0.1 \text { en faveur } \\
\text { du post-test }\end{array}$} & \multirow{2}{*}{0.98} \\
\hline Post-test & & 23.8571 & 1.76806 & & & \\
\hline
\end{tabular}

$\mathrm{N}=$ nombre des étudiants

$\mathrm{MA}=$ moyenne arithmétique

$\mathrm{ET}=$ écart type

$\mathrm{T}=$ valeur de " $\mathrm{t}$ " test $\quad \mathrm{S}=$ significative $\quad \mathrm{NS}=$ non significative

$\mathrm{VI}=$ valeur de l'influence

Le tableau précédent a présenté les moyennes arithmétiques du pré-test et celle du post-test, l'écart type et la valeur de T. On remarque qu'il y a une différence significative entre la moyenne des notes du groupe expérimental au pré-test et celle au post-test d'orthographe lexicale en FLE en faveur du post-test. La valeur de T calculé est plus grande que celle du tableau au niveau de $p>0,01$. On remarque, également, que la valeur de l'influence est très élevée (0.98). Ce qui vérifie la vérité de la première hypothèse de la recherche :

"Il y a une différence statistiquement significative entre la moyenne des notes des étudiants du groupe expérimental au prépost/test d'orthographe lexicale en FLE chez. les étudiants de la $2^{\text {ème }}$ année de la faculté de pédagogie de Minia en faveur du post/test.»

Ces résultats constatent l'efficacité des exercices interactifs via l'internet pour remédier aux erreurs d'orthographe lexicale en FLE auprès les étudiants de la $2^{\text {ème }}$ année de la faculté de pédagogie de Minia.

Tableau 3 : La différence entre le pré-test et le post-test d'orthographe grammaticale en FLE du groupe expérimental

\begin{tabular}{c|c|c|c|c|c|c}
\hline \hline Test & $\mathrm{N}$ & $\mathrm{MA}$ & $\mathrm{ET}$ & $\mathrm{T}$ & S. ou N.S. & VI \\
\hline Pré-test & \multirow{2}{*}{35} & 8.5143 & 2.11954 & \multirow{2}{*}{64.073} & $\begin{array}{c}\text { Significative au degré } \\
0.01 \text { en faveur du } \\
\text { post-test }\end{array}$ & 0.99 \\
\cline { 1 - 3 } Post-test & 23.4857 & 1.83706 & & \multicolumn{4}{c}{} \\
\hline
\end{tabular}


Le tableau précédent présente les moyennes arithmétiques du pré-test et celle du post-test, l'écart type et la valeur de T. On remarque qu'il y a une différence significative entre la moyenne des notes du groupe expérimental au pré-test et celle au post-test d'orthographe grammaticale en FLE en faveur du post-test. La valeur de $\mathrm{T}$ calculé est plus grande que celle du tableau au niveau de $\mathrm{p}$ > 0.01 . On remarque, également, que la valeur de l'influence est très élevée $\left(0.9^{9}\right)$. Ce qui vérifie la vérité de la deuxième hypothèse de la recherche :

"Il y a une différence statistiquement significative entre la moyenne des notes des étudiants du groupe expérimental au prépost/test d'orthographe grammaticale en FLE chez les étudiants de la $2^{\text {ème }}$ année de la faculté de pédagogie de Minia en faveur du post/test.»

Ces résultats indiquent l'efficacité des exercices interactifs via l'internet pour remédier aux erreurs d'orthographe grammaticale en FLE auprès les étudiants de la $2^{\text {ème }}$ année de la faculté de pédagogie de Minia.

Tableau 4 : La différence entre le pré-test et le post-test d'orthographe en FLE du groupe expérimental (Total)

\begin{tabular}{|c|c|c|c|c|c|c|}
\hline Test & $\mathrm{N}$ & $\overline{\mathrm{MA}}$ & ET & $\mathrm{T}$ & S. ou N.S. & $\overline{\mathrm{VI}}$ \\
\hline $\begin{array}{l}\text { Pré-test } \\
\text { (Total) }\end{array}$ & \multirow{2}{*}{35} & 17.9714 & 4.52760 & \multirow{2}{*}{62.576} & \multirow{2}{*}{$\begin{array}{c}\text { Significative au } \\
\text { degré } 0.01 \text { en faveur } \\
\text { du post-test }\end{array}$} & \multirow{2}{*}{0.99} \\
\hline $\begin{array}{l}\text { Post-test } \\
\text { (Total) }\end{array}$ & & 47.3429 & 2.97976 & & & \\
\hline
\end{tabular}

Le tableau précédent présente la moyenne arithmétique, l'écart type et la valeur de $\mathrm{T}$. On remarque qu'il y a une différence significative entre la moyenne des notes du groupe expérimental au pré-test et celle au post-test d'orthographe en FLE (Total) en faveur $\mathrm{du}$ post-test. La valeur de $\mathrm{T}$ calculé est plus grande que celle du tableau au niveau de $\mathrm{p}>0.01$. On remarque, également, que la valeur de l'influence est très élevée (0.99). Ce qui vérifie la vérité des deux hypothèses de la recherche et ce qui montre l'efficacité des exercices interactifs via l'internet pour remédier aux erreurs d'orthographe en FLE auprès les étudiants de la $2^{\text {ème }}$ année de la faculté de pédagogie de Minia. 


\section{Discussion des résultats :}

Les résultats de notre recherche ont montré l'efficacité des exercices interactifs via l'internet dans la remédiation aux erreurs communes d'orthographe lexicale et grammaticale en FLE auprès des étudiants de $2^{\text {ème }}$ année de la faculté de Pédagogie de Minia. Ces résultats se conforment avec les résultats de l'étude de Savchuk et al (2019) qui ont signalé que l'apprentissage interactif pousse les apprenants à s'engager de manière autonome dans le travail assisté par ordinateur. Nos résultats se conforment, également, avec les résultats de l'étude de Salem et Soleiman (2013) qui ont montré que les exercices interactifs via l'internet influencent positivement l'autonomie des apprenants de FLE ainsi que leur compréhension de cours de méthodologie de français.

Les exercices interactifs via l'internet ont permis aux participants de déterminer leurs erreurs d'orthographe, d'y réfléchir, de connaitre la forme correcte. Les exercices interactifs via l'internet présentent une rétroaction (simple ou descriptive) aux participants sur leurs erreurs d'orthographe. De même, les participants avaient la possibilité de refaire l'exercice et corriger leurs erreurs. Pour ces raisons, les participants ont admiré les exercices interactifs via l'internet, ils travaillaient régulièrement et sérieusement pour achever le travail demandé. Tous ces éléments ont contribué à rendre les exercices interactifs via l'internet efficaces pour remédier aux erreurs d'orthographe lexicale et grammaticale en FLE chez les étudiants de $2^{\text {ème }}$ année de la faculté de pédagogie de Minia.

Les erreurs remédiées par cette recherche en employant les exercices interactifs via l'internet sont de plusieurs types : les erreurs concernant les différentes manières d'écrire les sons de la langue qui sont considérés comme les bases essentielles de l'orthographe, les erreurs concernant les homophones, les erreurs concernant les lettres muettes, les erreurs concernant les consonnes doubles, les erreurs concernant le $\mathrm{H}$ aspiré, les erreurs de conjugaison des verbes, les erreurs de l'accord du participe passé, les erreurs concernant le pluriel $\mathrm{du}$ nom, les erreurs concernant les pronoms relatifs, les erreurs concernant le participe présent et le l'adjectif verbal et les erreurs 
concernant les prépositions. Notre expérimentation a traité plusieurs types d'erreurs d'orthographes à travers les exercices interactifs sur les mots et phrases. Des exercices interactifs de dictée ont été faits par les participants.

Notre recherche a vérfié l'efficacité des exercices interactifs via l'internet pour remédier aux erreurs communes d'orthographes en FLE. Ce résultat a ajouté une manière de remédier aux erreurs d'othographes ; cela est considéré comme un ajout aux moyens du traitement des erreurs d'orthographe vérifiés par les études antéieures: la carte mentale dans l'étude de Morenval, (2015), les dictées guidées dans les études de Le Brun et al (2016) et de Bosse (2019), les ateliers de négociation graphique dans l'étude de Mohamed (2020) ...

\section{Recommandations :}

A la lumière des résultats obtenus, la recherche actuelle recommande aux professeurs et aux enseignants du FLE d' :

- Utiliser les exercices interactifs via l'internet pour remédier aux erreurs d'orthographe lexicale en FLE ;

- Utiliser les exercices interactifs via l'internet pour remédier aux erreurs d'orthographe grammaticale en FLE ;

- Utiliser les exercices interactifs via l'internet pour enrichir le vocabulaire chez les apprenants du FLE ;

- Utiliser les exercices interactifs via l'internet pour enseigner les règles de grammaire du FLE ;

\section{Suggestions :}

Le chercheur suggère de :

- Vérifier l'efficacité des exercices interactifs pour favoriser la motivation des apprenants du FLE ;

- Vérifier l'efficacité de différents types de rétroactions pour remédier aux erreurs d'orthographe en FLE. 
Références :

Académie de Poitiers (2015). LearningApps, Espace pédagogique. Reçu de ww2.ac-poitiers ffrl danel IMG/ pdf/ tutoriellear ningapps-3.pdf

Académie de Rennes (2017). SOCRATIVE, un outil de questionnaire en ligne simple. https://www.toutatice.fr/portail/share/EH3LC5

Asselin, C., McLaughlin, A. (1992). Les erreurs linguistiques rencontrées dans les écrits des étudiants universitaires : analyse et conséquences. Revue de l'Association canadienne de linguistique appliquée, 14-1, p. 13-30.

Bosse, M.-L. (2019). Dictée guidée: un nouveau moyen de progresser en orthographe? Reçu de https://theconversation.com/dictee-guidee-un-nouveaumoyen-de-progresser-en-orthographe-107035

Bouheraoua, Z. (2006). Les exercices interactifs : outil d'apprentissage ? Actes du Colloque «Innovations, Usages, Réseaux », Nov 2006, Montpellier, France. edutice-00133651

Le Brun, I.; Bosse, M.-L. et Valdois, S. (2016). Améliorer l'orthographe chez les élèves de 10 à 13 ans : entrainement par dictées guides. Nouveaux Cahiers de la Recherche en Education, Volume 19, numéro 3, 2016, p.55-71.

Catach, N. (1995). L'orthographe française : traité théorique et pratique avec des travaux d'application et leurs corrigés. Paris : Nathan.

Catach, N. (2011). L'orthographe. Paris : Presses universitaires de France.

Chovino, L. et Dallaire, F. (2009). Études sur les stratégies pour accroître l'interactivité des cours en ligne - design et mise en cuvre. Le Réseau d'enseignement francophone à distance du Canada, Montréal.

Couzon, N. (2018). Les dictées guidées pour améliorer l'orthographe des élèves. http://rire.ctreq.qc.ca/2018/11/les-dicteesguidees-pour-ameliorer-lorthographe-des-eleves/ 
Demnard, D. (1981). Dictionnaire d'histoire de l'enseignement. Paris : Delarge.Le Dictionnaire La Rousse (en ligne). https://www.larousse.fr/dictionnaires/francais/orthograp $\underline{\text { he/56605 }}$

Fayol, M. et Jaffré, J. P. (2014). L'orthographe. Paris : Presses universitaires de France.

El Hilali, G. J. ; Nadeau, M. et Fisher, C. (2019). L'effet des dictées métacognitives-interactives sur la compétence à orthographier les homophones grammaticaux en rédaction », Repères [En ligne], $60 \mid 2019$, mis en ligne le 31 décembre 2019, consulté le 18 décembre 2020. URL : http://journals.openedition.org/reperes/2291

Fang, X. and Xue-mei, J. (2007). Error analysis and the EFL classroom teaching. US-China Education Review, Volume 4, No.9 (Serial No.34).

La Fondation Paul Gérin-Lajoie (2013). Recueil de dictées. https://fondationpgl.ca/wpcontent/uploads/2019/01/Recueil-dedicte\%CC\%81es.pdf

Gaafar, S. I. (2017). L'étude des erreurs grammaticales et orthographiques en expression écrite à la lueur de la conscience métalinguistique chez les étudiants au département de français à la faculté de pédagogie. Thèse de doctorat, faculté de pédagogie de Tanta.

Gilger, C. et Gervais, E. (2020). La Quizinière, pour créer simplement des activités numériques interactives. http://www.acgrenoble.fr/tice74/spip.php?article1409\&lang=fr

Henry, J.; Vandeput, Étienne \& Br. (2010). DENIS FAD : augmenter l'interactivitépour diminuer la distance ?, Ludovia. https://orbi.uliege.be/bitstream/2268/61868/1/Julie_Hen ry_Ludovia_2010.pdf

Ibrahim, M. G. R. (2016). Programme pour modifier les croyances des enseignants du FLE à propos de l'évaluation. Thèse de doctorat, faculté de pédagogie, université d'Ain Chams. 
Kennewell, S.; Tanner, H.; Jones, S. \& Beauchamp, G. (2008). Analysing the use of interactive technology to implement interactive teaching. Journal of computer assisted learning, Volume 24, Issue 1, February 2008, Pages 61-73.

Laigle, T. (2010). Concevoir et réaliser des exercices interactifs - Les outils (2). http://reseaupensant.net/post/Concevoir-etreacute $\% 3$ Baliser-des-exercices-interactifs-ndash\%3B-

Les-outils-\%282\%29

Mohamed, C. M. T. (2020). L'utilisation des ateliers de négociation graphique pour développer quelques compétences orthographiques chez les élèves du cycle primaire. Revue de l'Education (faculté de pédagogie de Sohag), Volume (77) Septembre, 2020.

Morenval, L. (2015). La carte mentale comme outil d'apprentissage en orthographe grammaticale: les homophones ont/on et l'accord dans le groupe nominal. Mémoire de maîtrise, Académie de Grenoble, Auvergne-Rhône-Alpes, France. https://dumas.ccsd.cnrs.fr/dumas-01205929/document

Mout, T. (2013). L'orthographe du français : usages et représentations d'adultes socio-différenciés. Approche pluridisciplinaire. THÈSE de Doctorat, Université de Grenoble.

Nurtika, A. (without date). Designing interactive classroom activities.

https://www.academia.edu/36695306/DESIGNING_IN TERACTIVE_CLASSROOM_ACTIVITIES

PragmaTICE (site internet). Educaplay. https://pragmatice.net/lesite/spip.php?article234

Salem, H. T. A. et Soleiman, R. I. M. (2013). Des exercices interactifs en ligne pour développer l'autonomie d'apprentissage et la compréhension de cours de la didactique du FLE. Revue des Etudes Arabes en Education et Psychologie, $\mathrm{N}^{\circ}$ 40, Partie 4, août, 2013.

Salem, H. T. A. et Soleiman, R. I. M. (2014). Les Erreurs D'orthographe Lexicale Et Grammaticale Dans Les Écrits Des Licenciés Département Du Français Ès 
Lettres, Et Ès Lettres Et Pédagogie : Étude Analytique Comparative. Revue des Etudes Arabes en Education et Psychologie, $\mathrm{N}^{\circ}$ 56, Décembre, 2014, p. 435-498.

Sautot, J. P. (2002). Raisonner l'orthographe au cycle 3. 2002. halshs-01267735.

Savchuk, N. M.; Sichkar, Svitlana A.; Khlystun, I. V.; Shuliak, S. A. \& Avramenko, V. I. (2019). The Interactivity of ICTin Language Teaching in the Context of Ukraine University Education. International Journal of Higher Education Vol. 8, No. 5; 2019. Published by Sciedu Press84ISSN 1927-6044E-ISSN 1927-6052

Shahar-Yames, D. and Share, D. L. (2008). Spelling as a selfteaching mechanism in orthographic learning. Journal of Research in Reading, Volume 31, Issue 1, 2008, pp $22-39$.

Sessoms, D. (2008). Interactive Instruction: Creating Interactive Learning Environments through Tomorrow's Teachers. International Journal of Technology in Teaching and Learning, $\mathrm{N}^{\circ}$ 4, 86-96.

Teye, V. (2011). Developing interactive learning system for teaching the elements of design in senior high schools. Thesis of Master, Faculty of Art, College of Art and Social Sciences, Kwame Nkrumah University of Science and Technology. Received from ir.knust.edu.gh/bitstream/123456789/3957/1/FINAL.pdf

Tuesday, O. S. et Temidayo, B. O. (2014). Interactionnisme médiatisé par l'internet et l'apprentissage du français langueetrangère: l'exemple des exercices grammaticaux. POLACJELF - Polac Journal of English, Linguistics and French, vo.1 no.2. Nigeria Police Academy, Wudil, Kano. 259 -273.

Wang, Y.-H. (2014). Use of interactive web-based exercises for english as a foreign language learning: learners' perceptions. Teaching English with Technology, 14(3), $16-29$. 